\title{
LoRaWAN IoT Technology for Energy Smart Metering Case Study Lebanon
}

\author{
Carine Zaraket ${ }^{1, \mathrm{a}^{\star}}$, Panagiotis Papageorgas ${ }^{2, \mathrm{a}}$, Michel Aillerie ${ }^{1, \mathrm{~b}}$ and Kyriakos \\ Agavanakis $^{2, b}$ \\ ${ }^{1}$ Université de Lorraine, CentraleSupélec, LMOPS, F-57070 Metz, France \\ 2 Rue E.Belin, F-57070 Metz Cedex, France \\ aCCorresponding author: carine.zaraket@univ-lorraine.fr \\ baillerie5@univ-lorraine.fr \\ ${ }^{2}$ Department of Electrical and Electronics Engineering, University of West Attica, P. Ralli \& Thivon \\ 250, 12244, Athens, Greece \\ a ppapag@uniwa.gr \\ bk.agavanakis@uniwa.gr
}

Keywords- LPWAN, LoRaWAN, Internet of Things, Energy Smart Metering

\begin{abstract}
Internet of things (IoT) technology is based on connecting each real object to the internet. Every single object is uniquely recognized and reachable over the network. IoT last mile connectivity is based on different communication technologies and protocols, where the majority is categorized as short-range networks that operate in ISM bands like Zigbee, Wi-Fi, and Bluetooth. Short-range technologies were successfully tested and deployed in different industrial sectors. However, in the energy sectors, the deployment is challenging in certain hard to reach areas where reliable last-mile connectivity is required between the home area network (HAN) smart meters and the meter data management system (MDMS). Therefore recently, Low Power Wide Area Network (LPWAN) technology implementations, which offer long-range connectivity, has emerged as a promising alternative for IoT in Energy applications. Within LPWAN, a variety of technology implementations exist and operate in licensed and unlicensed spectrum respectively like NB-IoT, LoRaWAN, and Sigfox.

In this article, we discuss both the performance of LoRaWAN in a real-world environment and its deployment as a low-cost, long-range, and reliable last-mile solution for energy smart metering in urban area scenario where a short-range solution may not be the optimum one. Furthermore, a prototype that is adapted to the existing Lebanese traditional energy sector was developed to test LoRaWAN's usefulness in Lebanon.
\end{abstract}

\section{Introduction}

With the growing energy demand, the energy generation distribution, the integration of renewables, and the enhancements achieved in IoT networks which target the Building Management systems (BMS) energy sector including outage management, the direction was to migrate the traditional grid to be smarter and more efficient. The smart grid is the integration of IoT networking technologies in the conventional electrical grid[1]. IoT networks introduce smart meters that permit real-time managing and monitoring of different parameters including the user's energy consumption. Building a smart grid is based on introducing and integrating a variety of smart technologies including smart meters, smart sensors, and different communication protocols. As a consequence, the global smart grid technology consists of groups of individual technologies that cover the whole grid from the generation to the transmission and distribution[2]. In developed countries, Smart grids have an essential role in the deployment of the electrical system by enabling low-cost solution deployment and more powerful performance[3,4]. With a smart grid the small electrical systems, that are not 
connected to the central system and deployed as a cost-effective solution for the electrification of rural area, can be later connected to the national central electricity system simply and easily.

In Lebanon, the first hands-on experience with IoT technology on a pilot basis has been demonstrated in the agriculture sector by some private companies, where LoRa sensors have been used for sensing the soil moisture[5]. Concerning the energy sector, the Lebanese power system "Electricté du Liban" (EDL) is using traditional energy meters based on electromechanical sensors, and energy consumption measurements are being captured monthly by the EDL employees. So far in the Utility sector, deploying IoT smart metering technologies depends on different requirements, like the country's geographical nature, the consumer's attitude for accessing their consumption data, the cost of a new smart meter ant the replacement cost of the existing system, and the lack of adoption of suitable standards and technologies that must be used to achieve an end to end connectivity[6].

In this article, our purpose is to integrate the new IoT technology to the existing traditional energy meter's set up in developing countries like Lebanon, to accelerate the communication between the meter and the utility in near real-time this is by including the Automated Meter Reading (AMR) infrastructure and to provide the consumer with a feedback of its real-time energy consumption. So we expect that our proposed solution will illustrate the smart meter functionalities by operating independently and transmitting data to the utility using LoRa technology as last-mile connectivity on a near real-time basis. The challenge that exists in such a setup with the present energy infrastructure in Lebanon is the variety of energy providers. The Lebanese energy market relies on both diesel generators and utility[6] so the setup should be able to take both energy measurements and to transmit it to two different providers, in addition to the integration of such system with the existing traditional meters. Hence we have tried to develop a low power, long-range connectivity and low-cost solution therefore, the direction was to the use of LPWAN technology[7] that is lately very well used for smart metering solutions while keeping untouched the present metering protocols. LPWAN technologies provide the utility with the possibility to read the metering data over a longer range than conventional solutions in an urban area as we have proved in our prototype for energy smart metering setup. Furthermore, in this article, we present an open-source and lowcost technology enablement of energy smart metering architecture based on LoRa with the use of a Microcontroller (MCU) PLATFORM (Arduino MeGa 25600). LoRa is the Physical layer of the LPWAN technology and it works in Unlicensed ISM bands (868 MHz in Europe, $915 \mathrm{MHz}$ in North America, and $433 \mathrm{MHz}$ in Asia). It is a low-cost technology that can be used for the interconnection of energy meters and sending the energy consumption data to the cloud via a Gateway. It provides a bidirectional wireless communication that is essential for implementing the metering infrastructure (AMR) in a Smart Grid. The structure of the presented study is as follows: Section II gives an overview of the metering protocols and the LoRa wireless technology, in section III the subsystems of the suggested prototype are presented, in section IV the prototype setup deployment procedure and experimental results are analyzed while in section $\mathrm{V}$ an overall summary of the presented work is given.

\section{Background}

With the latest improvements in IoT networks, globally utilities are installing smarts meters with different communication technologies. Lately, LPWAN technologies are considerably used and deployed in today's scenarios as a long-range solution for smart metering. Usually, with a smart module that is integrated into the energy meter, the majority of smart meter functionalities are achieved. The transmission of data utilizes low power, long-range, and narrowband transmission which will lead to a better stable and reliable network in addition to a low cost of implementation. This smart module could be programmed in a way to provide the utility and the consumer with 
notifications at predefined intervals of time. This section presents a detailed background of LoRa technology and the relevant metering technologies.

LPWAN technologies take place when other technologies (like Zigbee, WiFi, and Bluetooth) aren't good enough to fill all gaps in some case studies and even fail to achieve long-range coverage and performance[8]. For cellular M2M networks, they are too expensive in terms of hardware and services and it consumes too much energy. Hence LPWAN technologies are perfect to be deployed for nodes that need to transmit a small amount of data over a long-range while saving the battery life[9]. The best two areas for deploying LPWAN technologies are:

- LPWAN technologies are a perfect replacement for cellular M2M technology in smart cities and buildings and other applications like smart grid, smart lighting.

- In an application where long battery life is required like smart agriculture and water metering.

Sigfox[10] and LoRa[11] are competitors in the LPWAN space, and both of them belong to the Unlicensed ISM bands. While they differ in the business model and the technology itself but they are too similar in terms of functionalities and both are used and implemented for IoT applications that require long-range and low power communication protocol. Sigfox, an implementation of the narrowband technology, can be deployed for long-range applications. It uses the BPSK (binary phase-shift keying) modulation in the uplink and Gaussian Frequency-Shift Keying (GFSK) in the downlink, it offers a data rate up to 100bps and transmits data up to $10 \mathrm{kms}$ in an urban area and up to $40 \mathrm{kms}$ in the rural region[12]. Sigfox endpoints are inexpensive but the base station that controls and manages the network is more complicated than the corresponding element in LoRaWAN. While LoRaWAN operates at a wider band. On the other hand, Narrowband-Internet of Things (NB-IoT) is a standard developed by the third-generation partnership project (3GPP), that uses cellular telecommunication bands to connect a vast range of nodes[13]. It is used for massive M2M and IoT applications that required an extended range of transmission with a low cost and low power for long battery life. It coexists in long term evolution (LTE) or Global System for Mobile (GSM) under licensed frequency.

LoRa is a strong modulation technique for long-range, low data rate, and long battery life (up to 10 years) wireless communication technology. It is patented from Semtech, and uses a chirped spread spectrum modulation for layer one or the physical layer for LoRaWAN. The chirped spread spectrum modulation decreases the interference impact on data transmission providing increased reliability. LoRa MAC layer was developed by the LoRa Alliance and forms the data link and network layer. The adaptive rate is one of LoRa features, and it can be modified accordingly with the chosen bandwidth. The transmission energy is defined with the selection of the optimum spreading factor. The LoRa transceivers that must be integrated with the smart meter are not expensive compared to other technologies. LoRaWAN uses the ISM frequency bands with a data rate that varies from $0.3 \mathrm{kbps}$ to $50 \mathrm{kbps}$ and transmits data within a $5 \mathrm{~km}$ range in the urban region and $20 \mathrm{~km}$ in a rural area with a payload size of 243 bytes. In Europe, the adopted frequency band is between $863 \mathrm{MHz}$ and $870 \mathrm{MHz}$, however, in the US the adopted frequency is $915 \mathrm{~Hz}$. LoRaWAN end devices operate in three different operation modes, namely $\mathrm{A}, \mathrm{B}$, and $\mathrm{C}$, with each mode providing different uplink and downlink capabilities and energy needs accordingly[14].

Class A nodes listen to incoming messages directly after the upload of some data and then they go back to sleep mode (batteries saving mode). The access mode in use is the ALOHA mode for uplink transmission, after the transmission the class A node listens for a reply within two downlink windows, therefore the node can be inactive for a time duration (low duty cycle) which will save the battery life. Once the uplink transmission is deciphered successfully by the gateway then the 
downlink traffic can be transmitted. Hence, class, A nodes are the lowest power consumption nodes with high latency in the transmission and the reception of packets.

Class B nodes: the gateway sends beacon messages to the end device that is used to synchronize time windows for listening. This beacon is used for additional downlink traffic without previous successful uplink transmission. Class B devices are nodes with average power consumption and low latency in transmitting and receiving unicast and multicast packets.

Class $\mathrm{C}$ devices are always in listening mode, therefore they are connected to a power supply. Class $\mathrm{C}$ nodes are nodes with high power consumption, with low latency in the transmission and the reception of unicast and multicast packets[15].

LoRa networks are typically following a star topology where the gateway is just a relay between the end node and the central server. The end node communicates with the gateway via LoRaWAN and the gateway communicates with the backend via the IP standard.

In the actual scenario, the existing Home area network (HAN) technologies that are well standardized and in use like Wifi, Zigbee, Bluetooth, and Z-Wave, have some challenges in terms of power consumption and connectivity coverage. They belong to the short-range wireless communication technologies.

To expand their coverage limitation due to their physical short-range (less than $100 \mathrm{~m}$ ), they use mesh network topology. Hence, their major drawback is the high deployment cost to link the big number of nodes that are geographically scattered in a large area. Furthermore, since data is communicated via multi hops to the gateway, a big number of nodes are more loaded and congested than others which will impact their batteries' life (i.e. excessive use of energy), and therefore the network lifetime will be affected. Similar, the 2G, 3G, 4G, LTE cellular networks are developed to have a better traffic throughput, but they are not the best solution to be used for IoT applications due to battery consumption and they are proprietary to mobile operators.

On the other hand, the next-generation cellular proposal NB-IoT for IoT application is not yet welldeployed worldwide and specifically in Lebanon, therefore this gap is filled by LoRa technology. LoRa is developed to operate with IoT nodes which required a long battery life and low data transmission over a long distance.

Both short-range and cellular technologies are expensive solutions to be deployed in a vast area while LoRa is much simpler and presents a low-cost solution that is based on open standards.

LoRaWAN platforms are built according to the requirements and they are low-cost platforms. LoRaWAN fulfills the essential IoT requirements such as secure bi-directional communication, tracking services, and mobility. Furthermore, LoRaWAN specs afford transparent interoperability between smart things without a sophisticated implementation and provide the end-user, the developer, and the business with the right to enable the new rollout and solution with IoT.

\section{Proposed System Design and Architecture}

\section{Design constraints}

When designing a module to convert the traditional energy meter to be smart, there are many constraints and challenges to be taken into consideration and should be predefined. The most important challenge is to design an interoperable module that can be easily integrated with the existing infrastructure. This design has to read two different sources of energy (the Diesel and the Utility energy consumption). The following sections show the key points to be considered when designing such a system. 


\section{Design modularity and open-source}

In General, modular conception and open - source energy metering reading systems are for realizing traditional meters' migration with flexible functionality. This will assure the reliability, scalability, and modularity of the system to fit any additional new features.

Some challenges and needs should be defined before defining the hardware and software design of the system. The designed module in this paper is developed to add one of the smart features to the existing conventional meters. This system is user friendly and can be used by researchers to test LoRa in energy metering in traditional gird.

\section{Communication Protocol}

For the communication network usually, a smart meter has two network interfaces: The one connected to the Home area network (HAN) where the commonly used technologies are Zigbee and Z-wave. The second network interface, the Neighbor Area Network (NAN) for communicating with the Network Operator where power line communication PLC and LPWAN technologies are used. In this article, we focus on the NAN area, where the designed module is developed to provide to the Network Operator, the diesel generator provider, and the consumer/end-user the ability to read the energy consumption remotely in real-time and use them in a multitude of novel ways. Establishing a NAN communication link permits the meter to communicate with other meters and the utility by using different types of existing communication protocols in our case it is LoRa. Moreover, this module aims to transmit the energy consumption data via LoRa to an open-source cloud server where it can be accessible and can be operated on-premises by the different parties for energy trading applications.

\section{Materials characteristics}

Before selecting the components that will be used in our module, a commercial comparison in terms of cost and performance of the existing similar components is required. The most significant technical specs of electronic components for the performance evaluation contains accuracy, response time, and sensitivity. In particular, this applies to the $\mathrm{MH}$-sensor-series and the MicroController Unit (MCU) platform. The MCU platform should be open-source, programmed with a simple programming language based on an open-source integrated development environment, and fulfill the requirements in terms of processing capabilities, having enough storage, and finally equipped with enough interfaces to connect to the LoRa shield and the MH-sensor. The MH sensor is an Infrared sensor for detecting the pulses emitted from almost any old or new smart meter and which are used for the energy measurement readings.

\section{Prototype design}

\section{Hardware Design}

The MH-sensor is selected as a method to count the utility energy consumption. This is because it is a line detector, therefore it is capable to count the number of disc rotations of the electromagnetic energy meter that is still used by the Lebanese Utility. It is a fast response sensor and it is compatible with our design. On the other hand, the relay is used to differentiate between the electricity of Lebanon EDL and the diesel generator. The Arduino Mega is used as a microcontroller unit (MCU) platform for the metering. The Arduino platform was adopted because it is open-source, friendly to use, and easy to be programmed with large support from the scientific community. For data transmission LoRa shield module, which is a low-cost platform that can be easily deployed with the Arduino platform and it is used for data transmission on $868 \mathrm{MHz}$ frequency between the $\mathrm{MC}$ and the IoT server (Fig 1.). 


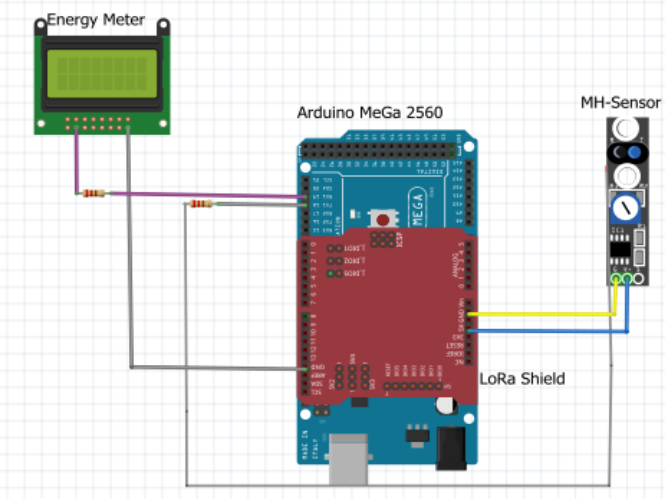

Fig. 1. Schematic Hardware Diagram

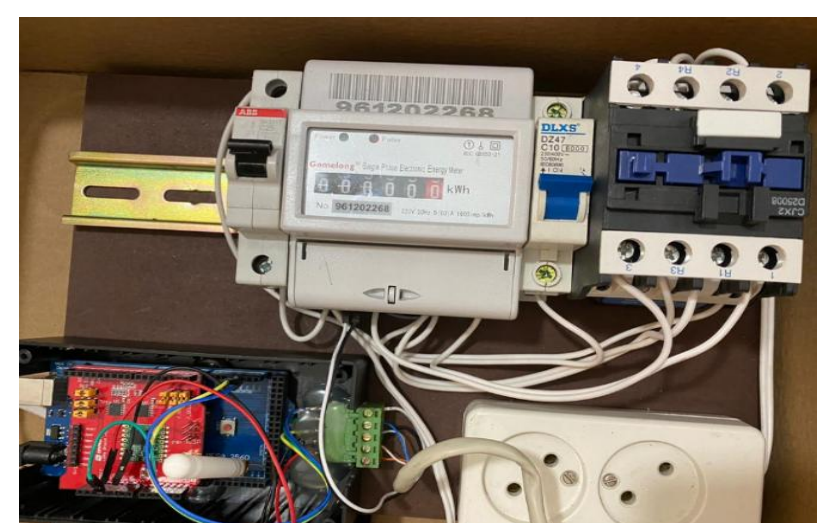

Fig. 2. Hardware Design

LoRa Prototype Components In our developed system, in order to read the energy consumption of both resources, the Diesel energy meter and the electricity of Lebanon (EDL) energy meter, we have integrated a loRa module which is composed of two main components: a microcontroller connected to a LoRa shield to retrieve the energy consumption measure from the meters and transfer this data trough loRa gateway to the network server as per the above architecture (Fig. 3). The microcontroller is the intelligent part of our whole module as it is configured to control all the components to perform and operate accordingly.

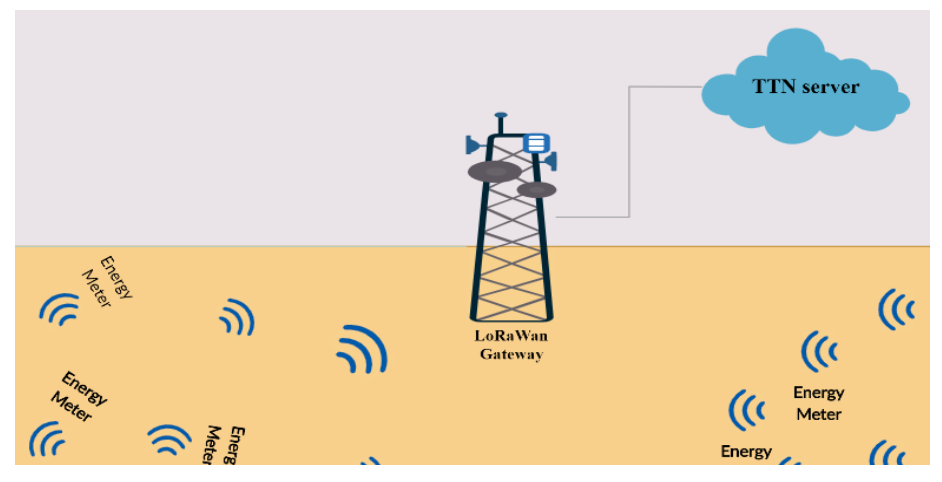

Fig. 3. LoRa Architecture.

In this prototype, the end device that is connected to the energy meter is composed of an Arduino MeGa microcontroller platform which integrates a LoRa shield for data transmission over a LoRaWAN network. This implementation is depicted in Fig. 4 where the LoRa shield is interfaced with the Arduino MeGa microcontroller's GPIO pins[16].

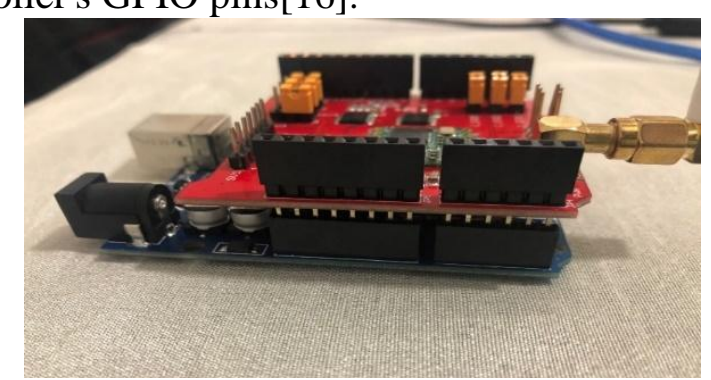

Fig. 4. Smart meter LoRa End Node

The LoRa Gateway is composed of an iC880A-SPI concentrator board[17]. This platform integrates $\mathrm{WiFi}$ and Ethernet connectivity as well as adequate memory resources for the microprocessor that runs a Linux distribution with $16 \mathrm{MB}$ Flash and $64 \mathrm{MB}$ of RAM and is integrated with a Raspberry Pi, an Antenna (Fig. 5). 


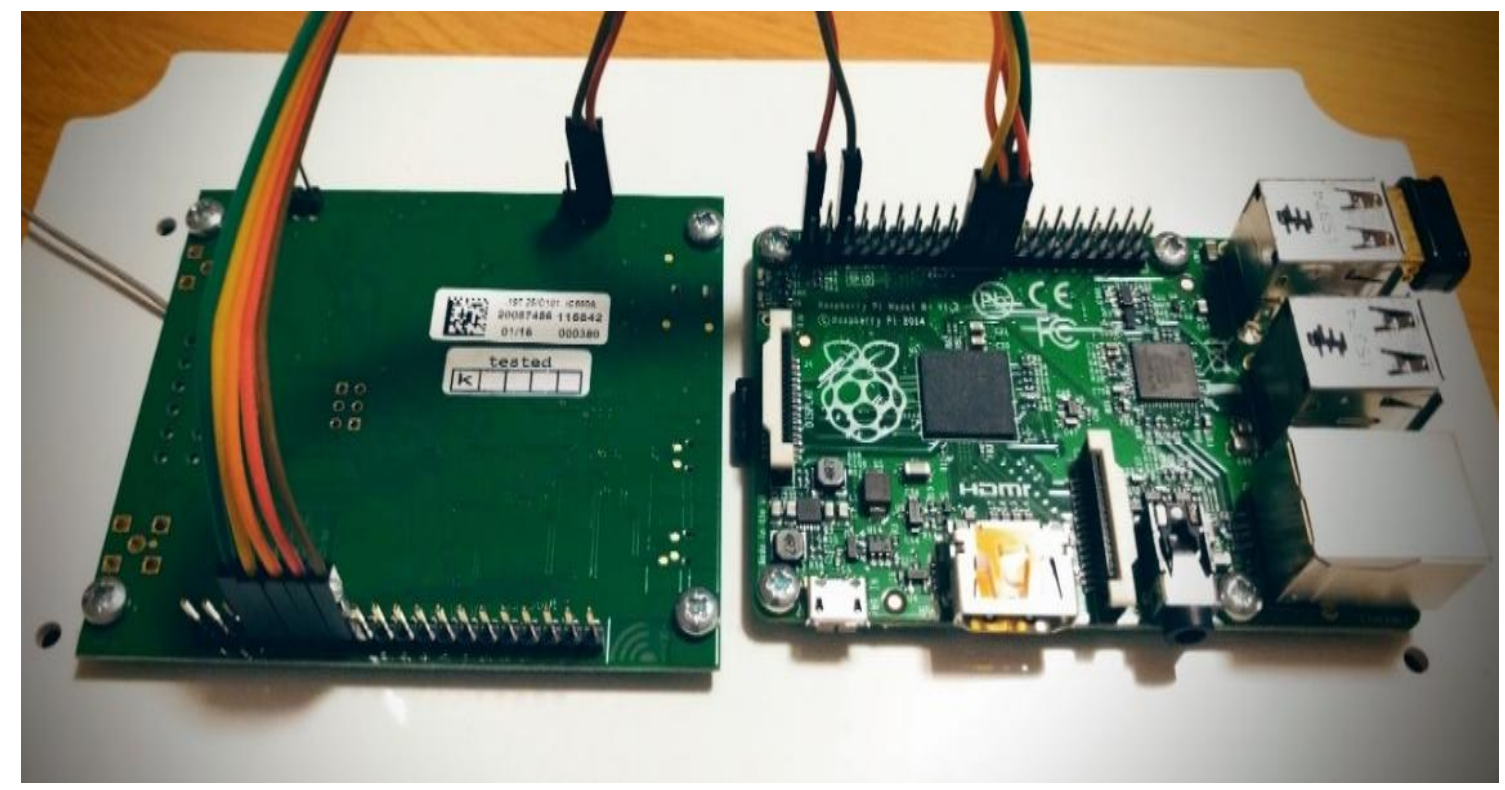

Fig. 5. The LoRa Gateway

LoRaWAN architecture is based on star-of-stars topology where the gateway plays only the role of a relay to transmit the data between the end device and the network server. The gateway is connected to the network server via the internet while the end-node is connected to the gateway via LoRa. Generally, end nodes communication is bi-directional, and all also support multicast communication for firmware upgrades over the air. The communication between LoRa node and the gateway can be performed using different frequencies and bandwidth.

\section{Connecting nodes to a LoRaWAN Network and Data Encryption}

After a LoRa node is connected to a network, packets are encrypted using a user-supplied key[18] . The LoRaWAN uses AES128 for encryption and adds a frame counter to the packets, while the application payload is encrypted by the AppSKey and the whole packet, including the frame counter and the DevAddr, is signed by the NwkSKey. As the NwkSkey is only known to the node and the network server, the integrity of a packet can only be verified within the network where the device is registered.

\section{Design Implementation}

Hardware Implementation The schematic and the implemented hardware designs are represented in Fig. 1. and Fig. 2. respectively. As shown in Fig. 1. the diesel generator and the MH-sensor are connected to the MC. The Arduino platform is programmed using a simple code snippet. As presented in Fig. 2 the LoRa shield is connected to the Arduino platform. Both the LoRa shield and $\mathrm{MH}$-sensor are powered by the Arduino board, while the Arduino board is supplied through a Universal Serial Bus (USB) port.

In our experiment, the test is done using the commonly used energy meters deployed in the Lebanese market, the Gomelong energy meter which is deployed by the Diesel generator providers, and a line detector $\mathrm{MH}$-sensor-series to read the utility's electromechanical energy meter. Both are interfaced with the microcontroller module that will transmit the data to the sever network (control center) via LoRa through the gateway. The module is connected to the energy meter through an interfacing module and serial port connections. The Arduino MeGa 2560 is connected directly to the diesel generator energy counter and is responsible for the pulse detection and counting (1600 pulses 
equals to $1 \mathrm{~kW}$ ). However, on the other hand, the Arduino is connected to an Infrared detector that is responsible for counting the number of disc rotation of the electromechanical EDL energy consumption ( 300 pulses equals to $1 \mathrm{~kW}$ ). These data are then transmitted via the gateway through the internet to the TTN cloud sever (open-source server) where it will be treated and used either by the generator's owner, the utility, or the consumer. The initial setup of the MC requires a baud rate of 115200 bps to be configured and Rx, Tx pins for the communications. The data is received by the MCU chip on the RX pin and is transmitted to the LoRa gateway via the LoRa shield communication interface as is depicted in the architecture proposed below in Fig. 6.

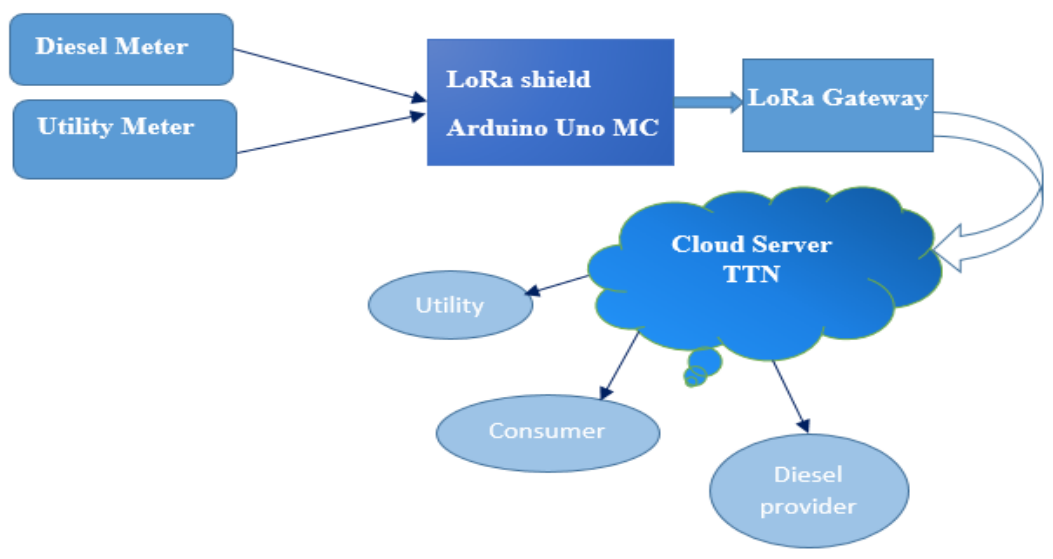

Fig. 6. LoRa module connected to energy Meters

\section{Software Implementation}

The developed code allows the MCU to count the number of pulses of each different source of energy and it sends a packet every $20 \mathrm{~ms}$ to the TTN server. This packet consists of 2 values the EDL and the diesel generator pulse number Fig. 7.

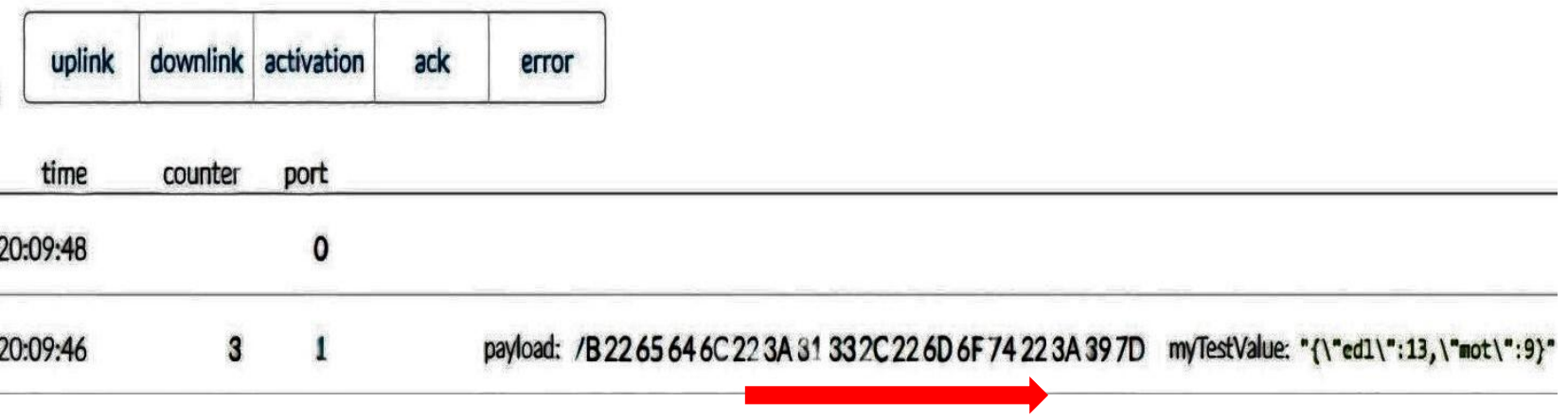

Fig. 7. The TTN Server dashboard Graphical User Interface (GUI)

\section{Results and Discussion}

\section{LoRa Performance Evaluation}

The proposed prototype is tested with the following variables:

In the beginning, LoRaWAN performance is tested over a real environment in Beirut, Lebanon to explore the limits of communication ranges in an outdoor environment. Our experiments are based on LoRa end node and LoRa gateway and the TTN server. 
The test was done under the following experimental conditions:

- Urban: Beirut City High density of users, buildings more than 4 floors

- GW Gateway, Effective Height $=25 \mathrm{~m}$

- Antenna Height $(20 \mathrm{~cm})$

- The implemented code sends one packet that contains the power consumption

- The transmission was tested at different distances away from the gateway.

The experimental results are illustrated below:

Time on air: Time on air is the time to transmit a packet of data. A Spreading Factor (SF) is specified for each transmitted packet of data, the SF is equal to SF $=\log 2$ (Rc/Rs), where the chip rate is Rc and the symbol rate is Rs. Therefore, there is a compromise between the communication range and SF. When the spreading factor is higher the date rate is lower, then the communication range is longer.

The below graph shows the time on-air with a bandwidth channel of $125 \mathrm{kHz}$ and a coding rate 4/5. However, as shown with a larger spreading factor the time on-air increases.

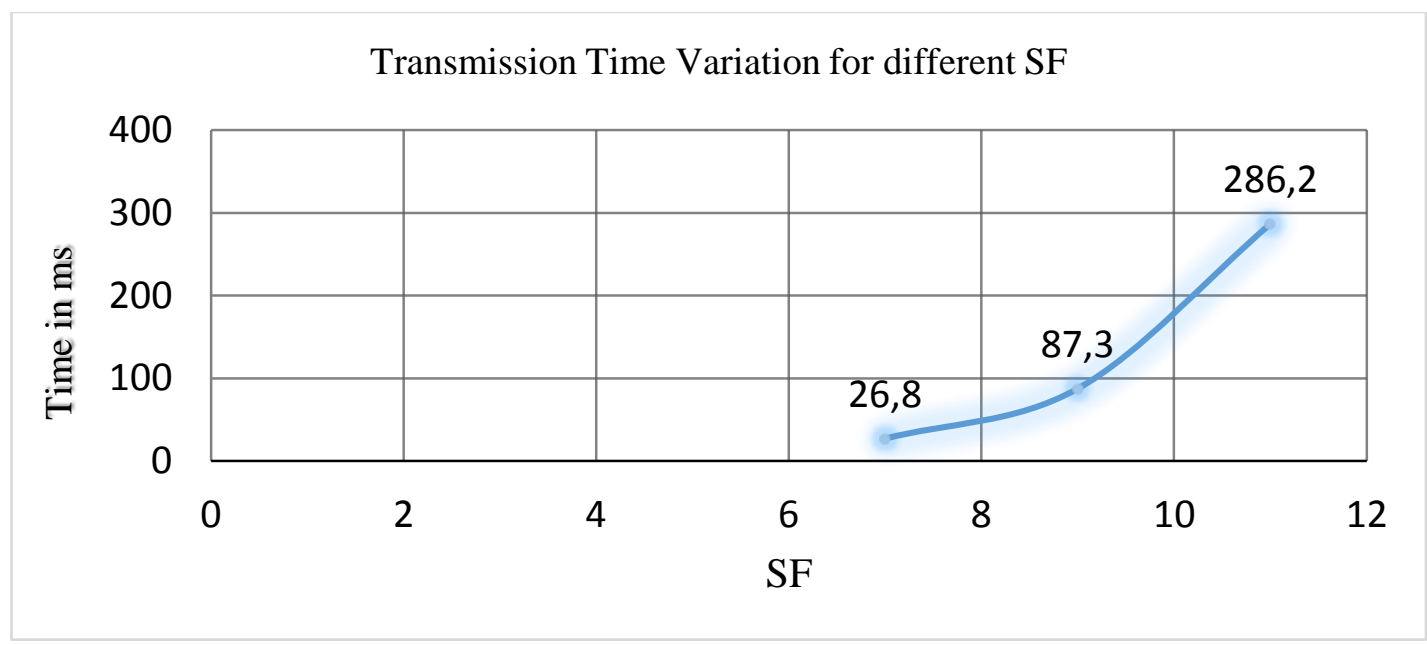

Fig. 8. Transmission Time Variation for different SF

Signal-to-Noise Ratio (SNR): first of all, the test was done in an outdoor environment with a line of sight nearby Beirut city. Fig. 9 shows the SNR values (in $\mathrm{dB}$ ) at each distance with periodical packet transmission. The SNR decreases when the distance from the gateway increase.

The SNR is the ratio between the received power signal and the noise level power level. The noise level depends on all the undesired interfering signal sources which will have an impact on the transmitted signal and will cause signal corruption which will lead to data packet retransmission.

- If the SNR value is positive, the received signal works above the noise level.

- If the SNR value is negative, the received signal works below the noise level.

Usually, the noise level is the physical limit of sensitivity, but LoRa performs under the noise level. Particularly LoRa SNR varies between: $-20 \mathrm{~dB}$ and $+10 \mathrm{~dB}$. If the SNR rate is close to +10dB which 
means that the signal at the reception is less corrupted. Signals with SNR value $-7.5 \mathrm{~dB}$ to $-20 \mathrm{~dB}$ under the noise level can be demodulated by LoRa [15].

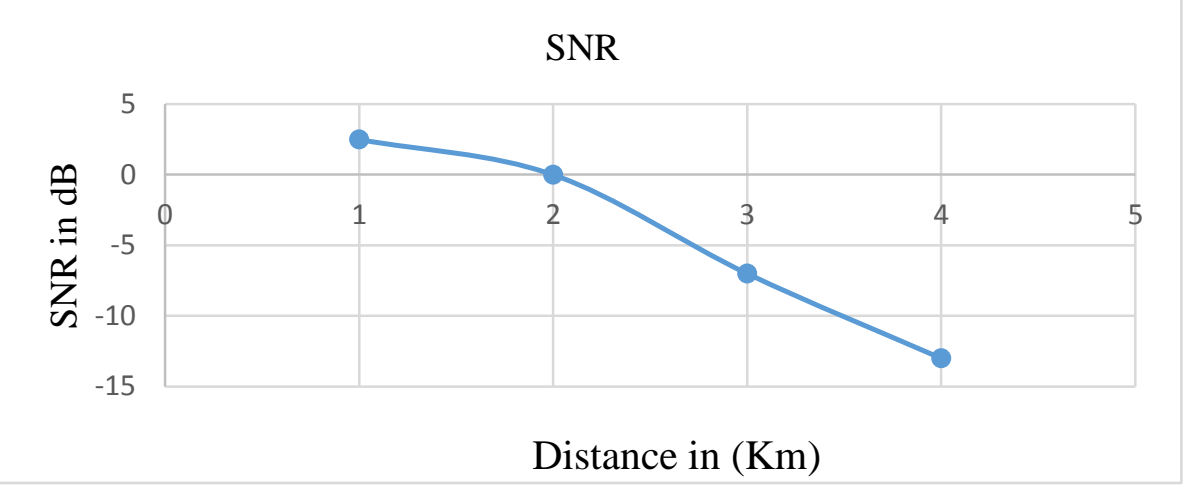

Fig. 9. SNR variation

Refer to the measure taken when the distance away from the gateway increases the SNR decreases and as we can see at $3 \mathrm{Km}$ away from the gateway the SNR was $-7 \mathrm{~dB}$ however at $4 \mathrm{Km}$ it becomes $13 \mathrm{~dB}$.

The packet losses are about $0 \%$ at a distance of $1 \mathrm{~km}$ and $2 \%$ at $2 \mathrm{~km}$. The height of the end-device plays role in the number of packet losses. Also, we can notice that the number of packet losses increases remarkably when the distance is more than $4 \mathrm{~km}$ as per the below graph. Therefore the gateway cannot receive the data transmitted from the end device at a distance higher than $4 \mathrm{Km}$.

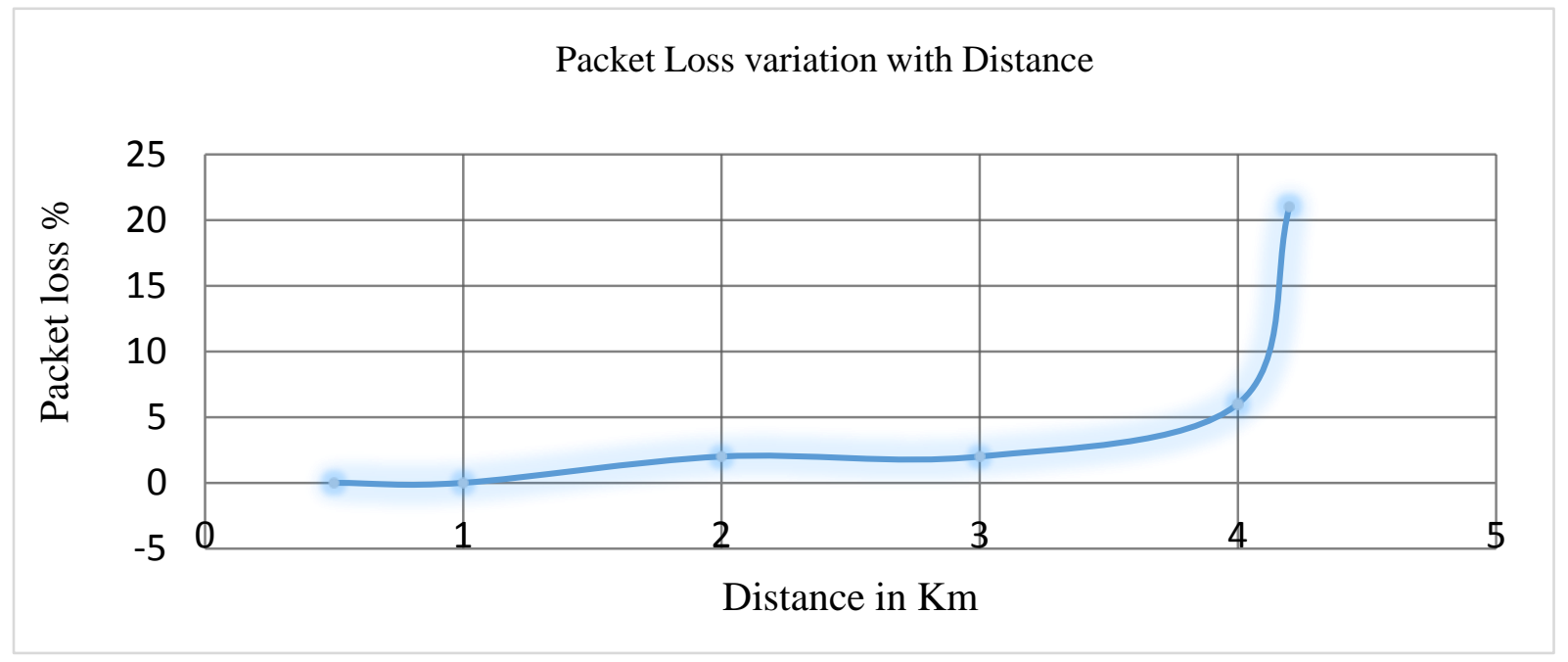

Fig. 10. Packet Loss variation with Distance

\section{Results Benchmarking}

The designed system for energy measurement readings was tested by connecting some home appliances to create an energy load on the system. The MC and the gateway are powered on and the gateway was online and connected to the TTN cloud server. On the other hand, the MH-Sensor was connected to the electromagnetic utility meter. The relay responsibility is to define which source of energy is on. The MC starts to count the number of pulses of the active source of energy, and then these computed measurements are communicated using the LoRaWan and the Gateway to the TTN server by using a specific code that contains information about the different Keys to establish a secure TLS connection. The transmitted data are continuously displayed as is depicted in Fig. 11 using the TTN dashboard GUI. 


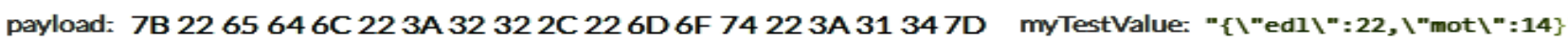

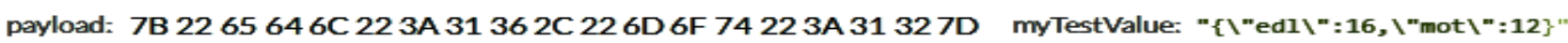
.

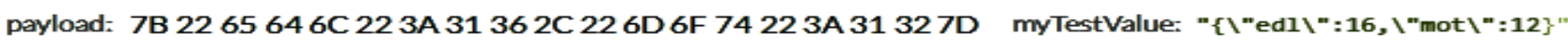
Paylo

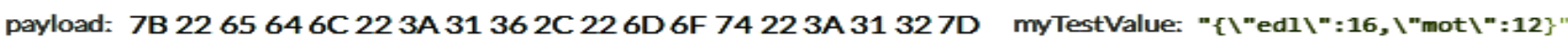

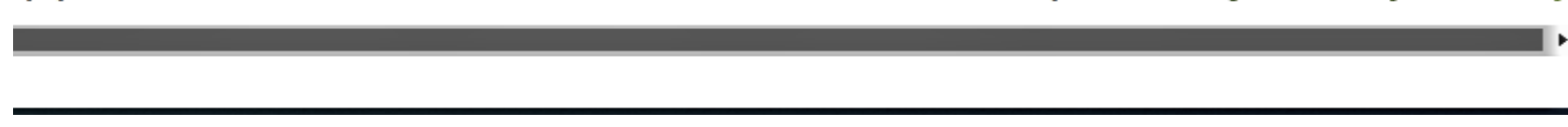

Fig. 11. Data Display

There is a small delay between the Arduino platform serial interface generated data packets and the values published on the TTN server, and this delay refers to the time taken by the LoRa shield to transmit the data packet and the cumulated delays from the TTN cloud server. This delay is not deterministic but as is in the order of seconds it is considered not important by considering that the directives in the European Union for energy smart metering demand sampling periods in the order of $15 \mathrm{~min}$.

To verify the setup we have connected specific electric loads in the IoT smart meter prototype and we have verified the validity of data between the recorded locally and the reported using the TTN cloud server during a specific time interval.

\section{Conclusions}

To summarize, this article proposes the design and deployment of an open-source, low-cost, and modular system based on LoRa telecommunication technology for energy metering applications. This solution was designed and proposed for the Lebanese market as an important solution for accessing the electricity outages by deploying a near real-time energy metering infrastructure. The design was deployed and tested in an experimental energy metering application, where the measured values were published to a cloud server, allowing for running analytics and be accessible from the Energy sector operators and the smart meter owners simultaneously. These values can be accessed, processed, and presented using a multitude set of tools enabling the deployment of thirdparty valuable services like the implementation of Virtual Power Plants for Energy curtailment, the engagement of consumers for load-shifting and power consumption reduction through gamification, and the introduction of Distributed Ledger Technologies for peer-to-peer energy trading. The proposed platform design can be easily deployed for improving the Lebanese energy sector by changing the traditional role of consumers to prosumers (producers and consumers) since most of the consumers own diesel generators and can participate in an Internet of Energy implementation. It provides an open-source energy-smart metering solution requiring minimal intervention in an existing electrical installation with a very low budget that can be valuable to the scientific 
community and the Electricity companies for experimentation and evaluation of IoT smart metering technology implementations.

\section{References}

[1] "No Title." https://www.smart-energy.com/regional-news/north-america/russia-attacked-theus-power-grid-what-if-they-dont-stop/.

[2] L. Specification, "The authors reserve the right to change specifications without notice. LoRa Specification 2 NOTICE OF USE AND DISCLOSURE 5,” 2015.

[3] Vitiello, S, Vasiljevksa, J, Filiou, C, "Cost-benefit analyses \& state of play of smart metering deployment in the EU-27," 2014.

[4] S. Ghosh, M. Pipattanasomporn, and S. Rahman, "Technology deployment status of U.S. smart Grid projects - Electric distribution systems," 2013 IEEE PES Innov. Smart Grid Technol. Conf. ISGT 2013, 2013, doi: 10.1109/ISGT.2013.6497867.

[5] "No Title," [Online]. Available: https://internetofbusiness.com/chateau-kefraya-cultivatessmart-vineyard-lebanon/.

[6] U. Nations and S. Division, "Technical assistance to Lebanon on improving energy statistics for sustainable development : Assessment mission report," no. July, 2019.

[7] LinkLabs, "Low Power, Wide Area Networks networks (LPWANs)," vol. 19, no. 2, pp. 855873, 2017.

[8] "No Title," [Online]. Available: https://www.iotworldtoday.com/2018/08/19/iotconnectivity-options-comparing-short-long-range-technologies/.

[9] R. S. Sinha, Y. Wei, and S. H. Hwang, "A survey on LPWA technology: LoRa and NB-IoT," ICT Express, vol. 3, no. 1, pp. 14-21, 2017, doi: 10.1016/j.icte.2017.03.004.

[10] “No Title.” http://www.link-labs.com/blog/sigfox-vs-lora.

[11] "No Title," [Online]. Available: http://www.3glteinfo.com/lora/lora-advantages/.

[12] “No Title," [Online]. Available: http://www.link-labs.com/blog/sigfox-vs-lora.

[13] K. Mekki, E. Bajic, F. Chaxel, and F. Meyer, "Overview of Cellular LPWAN Technologies for IoT Deployment :," pp. 197-202, 2018.

[14] L. Germani, V. Mecarelli, G. Baruffa, L. Rugini, and F. Frescura, "An IoT architecture for continuous livestock monitoring using lora LPWAN," Electron., vol. 8, no. 12, 2019, doi: 10.3390/electronics8121435.

[15] "LoRa Documentation," 2019.

[16] "No Title," [Online]. Available: http://wiki.dragino.com/index.php?title=Use_Lora_Shield_\%2B_Arduino_set_up_a_Lora_N ode.

[17] “No Title,” [Online]. Available: https://www.instructables.com/Raspberry-Pi-LoRaWANGateway/.

[18] “No Title," [Online]. Available: https://arxiv.org/abs/1706.03086. 
